\title{
Coherent phonons imprinted into reflectivity oscillations of laser-excited Bi through electron-phonon coupling \\ D. Boschetto ${ }^{1}$, E. G. Gamaly ${ }^{2}$, A. V. Rode ${ }^{2}$, B. Luther-Davies ${ }^{2}$ \\ D. Glijer ${ }^{1}$, T. Garl ${ }^{1}$, O. Albert ${ }^{1}$, A. Rousse ${ }^{1}$, J. Etchepare ${ }^{1}$ \\ ${ }^{1}$ Laboratoire d'Optique Appliquée, ENSTA/Ecole Polytechmique, Chemin de la Huniere, Palaiseau, France \\ ${ }^{2}$ Laser Physics Centre, Research School of Physical Sciences and Engineering The Australian National University, Canberra, ACT 0200 Australia
}

\begin{abstract}
We show that the reflectivity of laser-excited solid relates to phonons, driven by thermal forces, through the electron-phonon coupling rate. Controlled excitation of phonons is available by the optimum combination of laser and material parameters.

(a) Optical Society of America

OCIS codes:
\end{abstract}

The experimental and theoretical studies of the reflectivity oscillations of the probe beam from the single-crystal of bismuth excited at the deposited energy density $2.7 \mathrm{~mJ} / \mathrm{cm}^{2}-6.7 \mathrm{~mJ} / \mathrm{cm}^{2}$ are presented. The reflectivity was measured by the pump-probe technique with the accuracy $10^{-5}$ and with timeresolution of 35 fs that allowed observing a novel feature - the initial sharp drop of the reflectivity. The reflectivity oscillates with the frequency of $\mathrm{A}_{1 \mathrm{~g}}$ phonon in $\mathrm{Bi}$ in agreement with earlier findings [1,3-7].

Our analysis establishes the direct link between reflectivity oscillations and atomic vibrations through the electron-phonon coupling rate that is proportional to the phonon's amplitude. The major force driving atomic motion in a laser-excited solid is thermal force proportional to the temperature gradients. It is demonstrated that DECP [1] and strain-dependent polarisation [2-4] are lesser parts of the external field effect on a solid than the thermal force in opaque medium. The proposed theory explains all experimentally observed features of transient reflectivity without any ad hoc assumptions. The response of a medium on the laser action describes by the dielectric function that depends on atomic displacement, $q$, on electron density, $n_{e}$, and on the electron-phonon momentum exchange rate, $v_{e-p h}$ :

$$
\varepsilon=\varepsilon_{r, p}(q)+\varepsilon_{r, m}\left(n_{e}, v_{e-p h}\right)+i \varepsilon_{1, m}\left(n_{e}, v_{e-p h}\right)(1)
$$

Reflectivity variations are calculated through the Fresnel formulae and the Drude-like dielectric function as follows:

$$
\Delta R=A_{1} \cdot \Delta \varepsilon_{r, p}+A_{2} \cdot \Delta n_{e} / n_{0, e}+A_{3} \cdot \Delta v_{e-p h} / v_{0, e-p h} \text { (2) }
$$

Here $A_{1}, A_{2}, A_{3}$ are constant coefficients expressed through known optical data for Bi [8-9]. $A_{1}$ and $A_{3}$ are negative for $\mathrm{Bi}$, while $A_{2}$ is positive. The polarization-related term estimates in the Placzek approximation [2]. The absorbed energy density [10] comprises, $2 A \cdot F\left(t_{p}\right) / l_{s}=(0.48-1.19) \times 10^{3} \mathrm{~J} / \mathrm{cm}^{3}$ for the laser fluence of $F\left(t_{p}\right)=(2.7-6.7) \mathrm{mJ} / \mathrm{cm}^{2}$ (absorption, $\mathrm{A}=0.258$, skin depth $1_{\mathrm{s}}=2.984 \times 10^{-6} \mathrm{~cm}$ ). The number density of electrons, excited to conduction band by the avalanche-like process, reaches to the end of the pulse $n_{e}\left(t_{p}\right) \approx 2 A F_{p}(t) / \Delta \cdot l_{s}=(2-5) \times 10^{22} \mathrm{~cm}^{-3}$. Thus, $n_{e} \propto T_{e}$. The perturbation in electron-phonon collision rate is presented with the help of kinetic theory [11] as function of lattice temperature and phonon's amplitude. The time-dependent electron, $T_{e}$, and lattice temperature, $T_{L}$, in the skin-layer has been calculated using 2-temperature approximation [12] and material parameters from [8,9]. Time-dependent amplitude of atomic vibrations has been obtained as solution of thermal force-driven equation for damped ( $\gamma$ is damping) harmonic oscillations. Time-dependent reflectivity is determined by laser and laser-excited material parameters without ad hoc assumptions as the following:

\footnotetext{
${ }^{1}$ E-mail: davide.boschetto@ensta.fr
} 


\section{JThD11.pdf}

$$
\begin{aligned}
& \Delta R=-A_{11} \cdot\left(I / 10^{11} W / \mathrm{cm}^{2}\right)+A_{22} \cdot\left(T_{e}(t) / T_{e, m}\right)-A_{33} \cdot\left(T_{L}(t) / T_{e, m}\right)- \\
& -A_{44} \cdot\left\{\left(T_{e}+T_{L}\right) / T_{e, m}\right\} e^{-\gamma t} \cdot \cos \left\{\left(\omega_{0}^{2}-\gamma^{2}\right)^{1 / 2} t-\varphi\right\}
\end{aligned}
$$

The calculated $\Delta R / R$ function at $6.7 \mathrm{~mJ} / \mathrm{cm}^{2}$ is presented at Fig. $1 \mathrm{in}$ a good fit to the experiments.

Summary: We show that the reflectivity of laser-excited solid relates to phonons, driven by thermal forces, through the electron-phonon coupling rate. Controlled excitation of phonons is available by the optimum combination of laser and material parameters.

\section{References}

[1]. Zeiger H. J., Vidal J., et al. “Theory for displacive excitation of coherent phonons.” PRB 45(2): 768-778, (1992)

[2]. Y.R. Shen \&N. Bloembergen, Theory of Stimulated Brillouin and Raman Scattering, Phys. Rev.137, 1787-1805 (1965)

[3]. G.A. Garret et al, Coherent THz phonons driven by Light Pulses and the Sb problem: What is the Mechanism? PRL, 77,3661-3664 (1996)

[4]. E.D. Murray et al, Effect of Lattice Anharmonicity on high-amplitude phonon dynamics in photo-excited Bismuth PRB, 72,060301-4 (R) (2005)

[5]. Cheng T. K., Brorson S. D., et al. "Impulsive excitation of coherent phonons observed in reflection in bismuth and antimony.” Appl. Phys. Lett. 57(10): 1004-1005. (1990)

[6]. Hase M., Kitajima M., et al. "Dynamics of coherent anharmonic phonons in bismuth using high density photoexcitation" PRL 88(6): 67401(1-4), (2002).

[7]. M. F. DeCamp, D.A. Reis, P.H. Bucksbaum, and R. Merlin, Dynamics and coherent control of high-amplitude optical phonons in Bismuth, Phys. Rev. Lett. 64, 092301 (2001).

[8]. American Institute of Physics Handbook, D.E. Gray, Ed., 3d edition, McGraw-Hill Book Company, New York [9]. Thomas Garl, Ellipsometry of Bismuth at room temperature (2006) (unpublished)

[10]. E. G. Gamaly, A. V. Rode, B. Luther-Davies, and V. T. Tikhonchuk, Ablation of solids by femtosecond lasers: ablation mechanism and ablation thresholds for metals and dielectrics, Phys. of Plasmas, 9, 949-957 (2002).

[11]. M. Ziman, “Electrons and Phonons”, Clarendon Press Oxford (1960).

[12]. M. I. Kaganov, I. M. Lifshitz and L.V. Tanatarov, Relaxation between Electrons and the Crystalline Lattice,Sov. Phys. JETP,4 (2),173, 1957

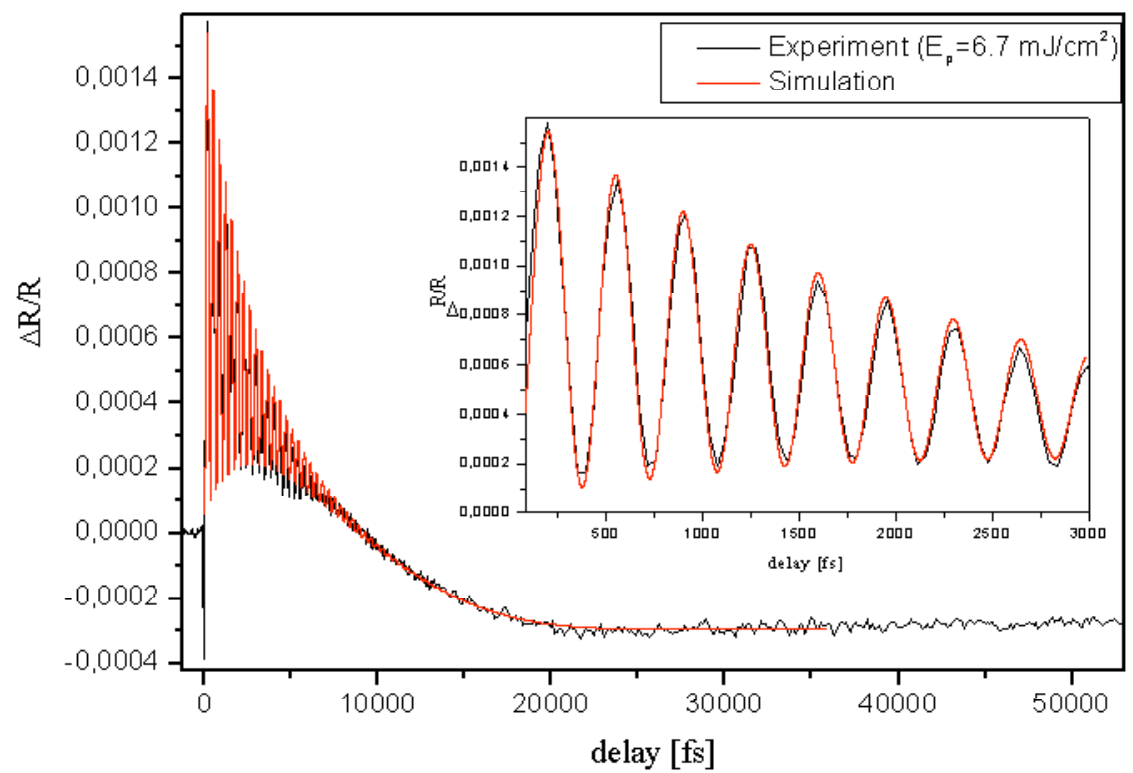

Fig.1The reflectivity variations as function of time-delay between pump and probe pulses 